\title{
Problem-Solving Based Intervention for Informal Caregivers: A Scoping Review
}

\author{
Quanwan Tao, Jun Zhang \\ School of Health Sciences, Wuhan University, Wuhan, China \\ Email: taoquanwan@foxmail.com
}

How to cite this paper: Tao, Q.W. and Zhang, J. (2019) Problem-Solving Based Intervention for Informal Caregivers: A Scoping Review. Open Journal of Nursing, 9, 951-971.

https://doi.org/10.4236/ojn.2019.99071

Received: August 6, 2019

Accepted: September 7, 2019

Published: September 10, 2019

Copyright $\odot 2019$ by author(s) and Scientific Research Publishing Inc. This work is licensed under the Creative Commons Attribution International License (CC BY 4.0).

http://creativecommons.org/licenses/by/4.0/

(c) (i) Open Access

\begin{abstract}
Background: Caregiving for someone is a huge task for informal caregivers. The caregiving role is often challenging and overwhelming for them. Stress, burden, and depression have been frequently identified as the negative caregiving experiences. This systematic review synthesized the available evidence for the problem-based intervention provided for caregivers as there is little insight about the effects of problem-based intervention on caregivers. Objectives: To describe: 1) the design of problem-solving intervention; 2) the effects of problem-solving intervention for caregiver outcomes. Methods: This review followed Arksey and O'Malley's methodological framework for conducting scoping reviews which entail setting research questions, selecting relevant studies, charting the data and synthesizing the results. FPRISMA guidelines were adopted and several databases were searched including MEDLINE; EMBASE; web of science. This review contains literature from 2012 to 2019 on problem-solving-based intervention which intended to caregivers. Results: 41 publications representing 27 unique problem-based interventions. Problem-solving-based intervention has different extent effects on caregiver emotion status, burden and quality of life. Conclusions: Problem-solving intervention is promising in caregiver intervention which could reduce caregiver depression, anxiety and burden.
\end{abstract}

\section{Keywords}

Informal Caregiver, Problem-Solving, Scoping Review

\section{Introduction}

Caregivers play a crucial role in supporting ill, disabled or older people. However, being a caregiver is challenging and overwhelming so that many caregivers feel unprepared for their role [1]. Moreover, caregivers are usually at higher risk 
for poorer physical and mental health as compared to their non-caregiving counterparts [2] [3] [4]. The global prevalence of depression and anxiety is $40.2 \%, 21.4 \%$ among stroke caregivers, respectively [5]. Caregivers of other conditions also have the varying extent of depressive symptoms and anxiety symptoms [4] [5] [6]. In addition, Caregivers experience substantial physical, financial, and psychosocial burden, as a result of providing help to their care recipients [7] [8].

Fortunately, there are interventions that try to alleviate the harms of caregiving outcomes. Growing evidence has demonstrated the benefit of problem-solvingbased intervention [9]. Problem-solving intervention is a self-directed process that aims at identifying possible solutions for specific problems encountered in one's daily life. [10] [11]. This approach is compromised by the following steps, problem definition and formulation, generation of alternative strategies, decision making and implementation. After practicing this step, caregivers tend to employ positive coping styles and have more problem-solving abilities which contribute to positive caregiver outcomes [12].

Efforts have been made to review the effect of problem-solving intervention on patients [13], there is still a gap in reviewing problem-solving intervention targeted at caregivers. Scoping review usually aims to collect and critically analyses various research studies and look for gaps in existing literature [14]. Thus, this study aims to conduct a scoping review to identify the design of problemsolving intervention and the effects of problem-solving intervention on caregiver outcomes.

\section{Methods}

This study employs a scoping review following the guidance of Arksey and O'Malley's methodological framework [14]. This framework is helpful to contribute to the rigor and transparency of the scoping review methodology and helps to enhance the reliability of the findings [14]. The five steps outlined by Arskey and O'Malley's [14] framework are: 1) Identifying the research questions, 2) Identifying relevant studies, 3) Study selection, 4) Charting the data and, and

5) Collating, summarizing and reporting the results.

\subsection{Framework Stage 1: Identifying the Research Question}

The research questions of this scoping review were: 1) how have problem-based intervention for caregivers been developed and delivered? (e.g., design, content and delivery mechanism) 2) what's the effectiveness of problem-solving based intervention designed for caregiver with their health outcomes?

\subsection{Framework Stage 2: Identifying Relevant Studies}

Relevant studies were searched using EMBASE, CINAHL, Web of science from 1 January 2000 to 22 May 2019 for original articles. The literature search was conducted by searching terms of (Problem-solving therapy OR Problem solving 
OR Problem-focused OR Problem-solving skills OR Problem-solving training OR Problem-solving treatment OR PST) AND (caregiver OR carer OR informal caregiver OR family caregivers).

\subsection{Framework Stage 3: Study Selection}

Studies were included if they met the following inclusion criteria: 1) wrote in English; 2) undertook experimental design; 3) focused on caregivers of elderly people; 4) delivered a problem-solving based intervention. Studies were excluded: 1) studies focused on caregivers to pediatric patients; 2) studies focused on paid or formal caregivers; 3 ) unpublished dissertations, reviews, nonexperimental studies. Two reviewers independently conducted the searching and screening.

The searching identified 1000 literature for consideration. After duplications were eliminated, there are 565 abstracts left for further identification. Finally, after the abstract and full-text screening, forty-one papers representing 27 unique interventions were included in this review. A flow chart of the research search is displayed in Figure 1.

\subsection{Framework Stage 4: Charting the Data}

The key information relating to the intervention were systematically extracted the following information for each study: author; year of publication; country; study design; caregiver population characteristics (mean age, health professional occupation, hours of caregiving for informal caregivers, diagnoses for which the patient is receiving care); sample size; intervention and control (when applicable) characteristics (material covered and hours of instruction); and outcome measures (physiological and non-physiological).

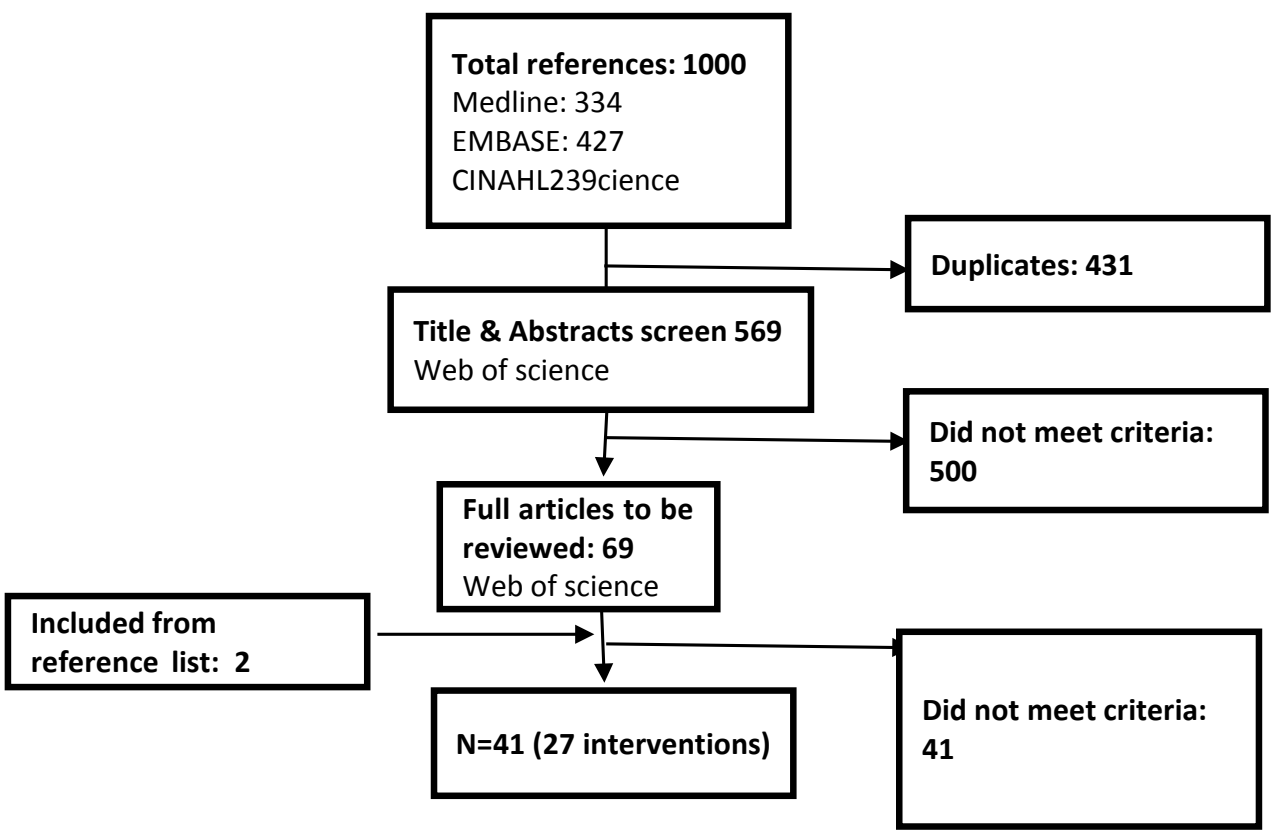

Figure 1. Flow chart of the search strategy. 


\subsection{Framework Stage 5: Collating, Summarizing and Reporting the Results}

This review employed a narrative synthesis of the included interventions to answer the research questions. Analysis of the studies was developed inductively, focusing on how THE problem-solving intervention on caregivers' outcomes. This data analysis was taken through an interactive process of combining, categorizing, summarizing and comparing information across studies. The summative findings are in accordance with the study questions. This study did not conduct a quality assessment or limit inclusion of studies based on their methodological rigor because including a wide range of literature are best-suited to get a more complete overview of the literature.

\section{Results}

\subsection{Results of the Search}

Problem-solving interventions which delivered to caregivers of any adult in need of care were screened in this review. Of the 27 interventions representing 41 articles, 24 employed randomized control design, two of them involved randomizing in blocks [15] [16]. Other interventions incorporated one group of before-after design [17], matched cohort design [18]-[22]. Samples used in the 27 interventions ranged from 6 [17] to 514 [23]. The selected studies included interventions targeting mainly on caregivers of people with dementia, stroke, traumatic brain injury, cancer and mental illness. Most of the interventions were delivered in USA, while the rest of interventions took place in Canada, Australia, Hongkong, and Iran.

These interventions target at caregivers of many kinds of condition, including caregivers of cancer $(\mathrm{N}=4 / 4)$, dementia $(\mathrm{N}=4 / 4)$, mental illness $(4 / 5)$, traumatic brain injury $(M=4 / 4)$ and others. Details of the intervention group are shown in Table 1. Most of the interventions a combination of face to face and telephone, while others used face to face, telephone, web alone. Few interventions delivered in group. Numbers of sessions ranged from 3 [23] [24] [25] to 12 [23] [24] [25]. Details about the intervention design are provided in Table 2.

\subsection{Problem Solving Intervention Effects on Caregiver Outcomes}

In accordance with the guideline of scoping review, this article did not appraise these interventions for methodological rigor. Therefore, the quality of studies is not available. The results displayed are a synthesis of what has been reported in preview studies. Nine kinds of results were reported

\subsubsection{Caregiver Mood Status}

Mood status for caregivers often reported with depression incidence, depression level, anxiety. Most problem-solving based intervention delivered to caregivers resulted in reduced depression level and anxiety level [16] [21] [23]-[32]. One RCT revealed that delayed incidence of depression [30]. 
Table 1. Study outcome measurements.

\begin{tabular}{|c|c|c|c|}
\hline $\begin{array}{l}\text { No. Intervention/ } \\
\text { No. Study }\end{array}$ & Outcome & Study & Positive results \\
\hline $16 / 27$ & Mood & $\begin{array}{l}\text { [37] problem-solving [23] [24] [25] [42] [43] problem-solving } \\
\text { [17] problem-solving [24] [28] [46] problem-solving } \\
\text { orientation [36] problem-solving [47] problem solving [22] [48] } \\
\text { problem solving [49]-[53] problem-solving ability [56] problem } \\
\text { solving [39] coping [18] coping }\end{array}$ & $\begin{array}{l}\text { [37] improved problem solving [17] } \\
\text { improved problem solving [24] [28] [46] } \\
\text { less negative problem orientation [47] } \\
\text { improved problem solving [56] better } \\
\text { problem-solving }\end{array}$ \\
\hline $11 / 16$ & Burden & $\begin{array}{l}\text { [37] Cancer-related distress [35] subjective and objective } \\
\text { burden [23] [24] [25] caregiver reaction [26] burden [13] [43] } \\
\text { burden [30] [32] distress, burden [18] perceived stress, burden } \\
\text { [33] burden [15] distress [36] burden [47] distress }\end{array}$ & $\begin{array}{l}{[37] \text { reduced distress [35] reduced }} \\
\text { burden; Remained low level distress [26] } \\
\text { lower burden [42] reduced burden [30] } \\
{[32] \text { lower leverl of distress and burden }} \\
\text { [33] reduced burden [15] decreased } \\
\text { distress [36] reduced burden }\end{array}$ \\
\hline $10 / 16$ & QOL & $\begin{array}{l}\text { [37] qol [22] [25] QOL [38] QOL [40] QOL [26] QOL [19] [20] } \\
\text { [21] fatigue, sleep quality [33] qol [15] QOL [49]-[53] health } \\
\text { complaints }\end{array}$ & $\begin{array}{l}\text { [23] enhanced QOL [19] [20] [21] } \\
\text { reduced fatigue [33] enhanced qol }\end{array}$ \\
\hline $10 / 20$ & Problem solving & [17] [24] [28] [37] [46] [47] [56] problem-solving & $\begin{array}{l}\text { [37] improved problem solving [17] } \\
\text { improved problem solving [24] [28] [46] } \\
\text { less negative problem orientation [47] } \\
\text { improved problem solving [56] better } \\
\text { problem-solving }\end{array}$ \\
\hline $6 / 8$ & $\begin{array}{l}\text { Sense of competence/ } \\
\text { self-mastery/efficacy/ } \\
\text { preparation }\end{array}$ & $\begin{array}{l}\text { / [16] sense of competence (protocol) [18] self-mastery, } \\
\text { (competency [31] sense of competence [19] [20] [21] } \\
\text { self-efficacy [48] caregiver preparedness [56] preparation }\end{array}$ & $\begin{array}{l}{[18] \text { improved mastery, competence }} \\
\text { [19] [20] [21] improvement in } \\
\text { self-efficacy [56] more prepared to } \\
\text { caregiving role }\end{array}$ \\
\hline $4 / 7$ & Life satisfaction & [49]-[53] life satisfaction [54] life satisfaction [55] & {$[49]-[55]$} \\
\hline $3 / 6$ & $\begin{array}{l}\text { Mutuality/family } \\
\text { function }\end{array}$ & $\begin{array}{l}\text { [37] quality of relationship [19] [20] [21] mutuality, [22] [48] } \\
\text { family function }\end{array}$ & [37] improvement in relationship \\
\hline $2 / 2$ & Knowledge and skill & $\begin{array}{l}\text { [40] knowledge and skill acquisition [41] medication } \\
\text { management }\end{array}$ & [41] reduced medication deficiencies \\
\hline $2 / 2$ & Coping & [39] coping [18] coping & $\begin{array}{l}\text { [39] Increased higher task-oriented } \\
\text { coping/emotion-oriented coping [18] } \\
\text { improved task-oriented coping }\end{array}$ \\
\hline $1 / 1$ & Personal control & [36] personal control & [36] increased personal control \\
\hline
\end{tabular}

\subsubsection{Caregiver Burden}

Although lack of consensus amongst studies on how problem-solving intervention affected caregiver burden, there are still significant resulted in caregiver burden after completion of intervention [15] [26] [30] [32]-[37]. Reductions in subjective and objective burden were observed in one study of dementia caregivers [35].

\subsubsection{Quality of Life}

Of all the interventions measuring quality of life, two interventions of 3 articles indicated that caregivers gained enhanced quality of life benefiting from the intervention [23] [33]. A problem-solving education intervention for caregivers of people received found better health outcomes, fatigue [19] [20] [21]. 
Table 2. Summary of articles included in the study.

\begin{tabular}{|c|c|c|c|c|c|c|c|c|}
\hline & $\begin{array}{l}\text { Author/ } \\
\text { year/study } \\
\text { location }\end{array}$ & $\begin{array}{c}\text { Method } \\
\text { (RCT/ } \\
\text { controlled/ } \\
\text { uncontrolled) }\end{array}$ & Aim & $\begin{array}{l}\text { Characteristic } \\
\text { of caregiver \& } \\
\text { Care recipient }\end{array}$ & $\begin{array}{l}\text { Intervention } \\
\text { Typology } \\
\text { (group/ } \\
\text { individual) }\end{array}$ & $\begin{array}{l}\text { Program } \\
\text { length, } \\
\text { number, } \\
\text { duration }\end{array}$ & $\begin{array}{l}\text { Outcome and } \\
\text { instrument }\end{array}$ & Significant result \\
\hline 1 & $\begin{array}{l}{[37]} \\
\text { USA }\end{array}$ & RCT & $\begin{array}{l}\text { To test } \\
\text { The efficacy of } \\
\text { PST for } \\
\text { spouses of } \\
\text { men with } \\
\text { prostate } \\
\text { cancer. }\end{array}$ & $\begin{array}{l}\text { Spouse } \\
\text { f caregivers of } \\
\text { men with } \\
\text { prostate cancer } \\
N=164 \\
\text { IG }=78 \\
\text { CG }=86\end{array}$ & $\begin{array}{l}\text { Individual } \\
\text { face-to-face }\end{array}$ & $\begin{array}{l}\text { - Six to eight } \\
\text { sessions } \\
\text { - Delivered by } \\
\text { trained staff }\end{array}$ & $\begin{array}{l}\text { Timing: } \\
\text { - Preintervention } \\
\text { - Post-intervention } \\
\text { - 3-month post } \\
\text { intervention } \\
\text { follow-up } \\
\text { Outcome } \\
\text { measurement: } \\
\text { - Problem-solving } \\
\text { - Cancer-related } \\
\text { distress } \\
\text { - Mood states } \\
\text { - Quality of life } \\
\text { - Quality of romantic } \\
\text { relationships }\end{array}$ & $\begin{array}{l}\text { - Constructive } \\
\text { problem-solving } \\
\text { improved more for } \\
\text { intervention. } \\
\text { - Greater decrease in } \\
\text { cancer-related } \\
\text { distress for } \\
\text { intervention. } \\
\text { - Greater } \\
\text { improvement in } \\
\text { dyadic relationship } \\
\text { for intervention. }\end{array}$ \\
\hline 2 & $\begin{array}{l}{[35]} \\
\text { USA }\end{array}$ & RCT & $\begin{array}{l}\text { To examine } \\
\text { whether } \\
\text { problem- } \\
\text { solving } \\
\text { therapy (PST) } \\
\text { would reduce } \\
\text { burden levels } \\
\text { of caregivers }\end{array}$ & $\begin{array}{l}\text { Caregivers of } \\
\text { individuals } \\
\text { diagnosed with } \\
\text { mild cognitive } \\
\text { impairment } \\
\text { (MCI) or } \\
\text { early-stage } \\
\text { dementia (AD) } \\
\mathrm{N}=73 \\
\text { Intervention: } 36 \\
\text { Control: } 37\end{array}$ & $\begin{array}{l}\text { Individual } \\
\text { Face-to-face } \\
\text { and telephone } \\
\text { contact }\end{array}$ & $\begin{array}{l}\text { Nine-sessions } \\
\text { with two } \\
\text { phases. }\end{array}$ & $\begin{array}{l}\text { Timing: } \\
\text { - Preintervention } \\
\text { - One-month } \\
\text { post-intervention } \\
\text { - 3-months post } \\
\text { intervention } \\
\text { - 6-month post } \\
\text { intervention } \\
\text { - 12month post } \\
\text { intervention } \\
\text { Outcome } \\
\text { measurement: } \\
\text { - Subjective and } \\
\text { objective caregiver } \\
\text { burden }\end{array}$ & $\begin{array}{l}\text { - Caregivers reduced } \\
\text { burden levels in IG. } \\
\text { - Distress for } \\
\text { caregivers in IG } \\
\text { remained low over } \\
\text { time. }\end{array}$ \\
\hline $3 / 1$ & $\begin{array}{c}{[29]} \\
\text { Spain }\end{array}$ & RCT & $\begin{array}{l}\text { To evaluate } \\
\text { the } 8 \text {-year } \\
\text { effect of } \\
\text { indicated } \\
\text { Prevention of } \\
\text { depression in } \\
\text { female } \\
\text { caregivers. }\end{array}$ & $\begin{array}{l}\text { Non-professiona } \\
1 \text { female } \\
\text { caregivers with } \\
\text { subclinical } \\
\text { depressive } \\
\text { symptoms } \\
\mathrm{N}=173 \\
\mathrm{IG}=89 \\
\mathrm{CG}: 84\end{array}$ & $\begin{array}{l}\text { Four-six } \\
\text { participants in } \\
\text { one group } \\
\text { Face-to-face }\end{array}$ & $\begin{array}{l}\text { Five weekly } \\
\text { group sessions }\end{array}$ & $\begin{array}{l}\text { Timing: } \\
\text { - Preintervention } \\
\text { - One-month } \\
\text { post-intervention } \\
\text { - 1-months post } \\
\text { intervention } \\
\text { - 3-month post } \\
\text { intervention } \\
\text { - 6-month post } \\
\text { intervention } \\
\text { - 1-year follow-up } \\
\text { Outcome } \\
\text { measurement: } \\
\text { - Depression status } \\
\text { - Depressive symptom } \\
\text { severity }\end{array}$ & $\begin{array}{l}\text { Lower depressive } \\
\text { symptom severity } \\
\text { for caregivers in IG. }\end{array}$ \\
\hline
\end{tabular}




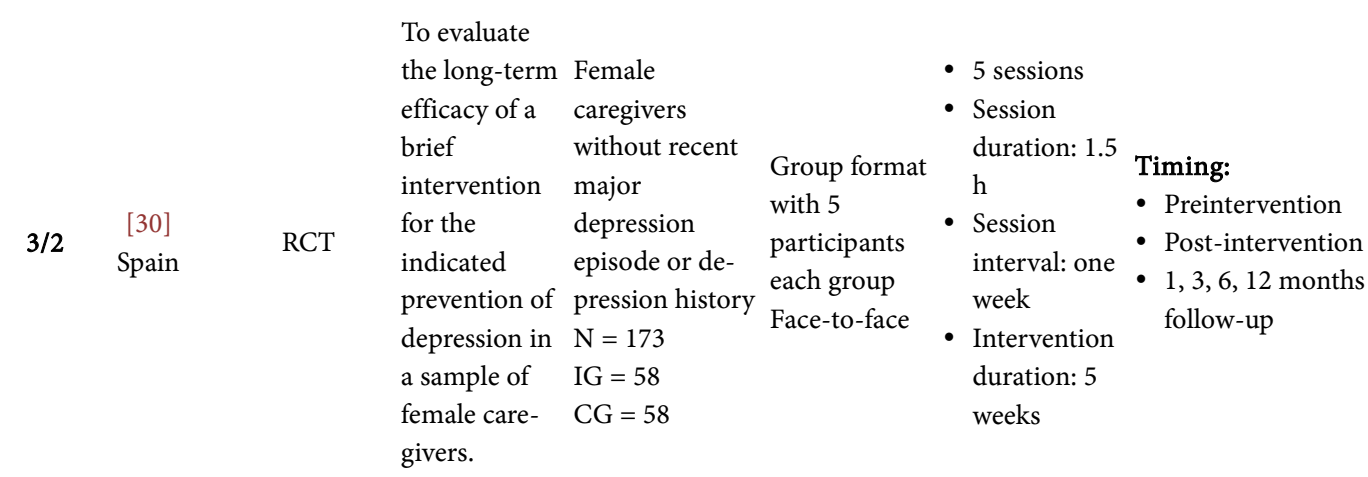

$3 / 3$
As above

As above
As above

As above
As above

As above
- At the 12-month follow-up, a lower incidence of depression was found in IG.

- Significant delay in the onset of depression in IG.

- Good complier caregivers had a lower incidence of depression

- Intervention effect on depressive symptoms, emotional distress and caregiver burden were maintained for 12 months

- At post-treatment, depression symptoms in ighadremitted significantly more than in CG, with a large effect size.

- IG exhibited a significantly greater reduction in emotional distress and caregiver burden than $\mathrm{CG}$.

To assess the impact of a problemsolving Hospice family intervention caregivers on caregiver $\mathrm{N}=514$ Face to face or 3 weekly sesvideoconfe- sions quality of life IG (face-to-face) rencing Duration: and anxiety, $=171$ Individual

Duration:

To compare its IG (videoconfeSession duraeffectiveness $\quad$ rencing $)=171 \quad$ Blinding delivered face $C G=172$ tion: $45 \mathrm{~min}$

to face and via videoconferencing.

To compare the effectiveness of a PST intervention delivered $\mathrm{N}=126$

4/2 [25] As above

face-to-face $=77 \quad$ As above As above As above

with one IG (videophone)

delivered via $=49$

videophone to

hospice primary

caregivers.
Timing:

- Preintervention

- Day 15

- Day 30

- Day 60

Outcome

measurement:

- Quality of life

- Anxiety

- Problem-solving

- Caregiver reaction (positive and negative reaction)
- Reduced anxiety and improved quality of life in IG

(face-to-face) compared with CG

- Videoconferencing mediated intervention was not as effective as the F2F group

- PST delivered via video was not inferior to face-to-face delivery.

- Caregiver quality of life improved and state anxiety decreased under both conditions. 


\section{Continued}

\begin{tabular}{|c|c|c|c|c|c|c|c|c|}
\hline $4 / 3$ & [24] & As above & As above & As above & As above & As above & As above & As above \\
\hline 5 & $\begin{array}{l}{[38]} \\
\text { USA }\end{array}$ & $\begin{array}{l}\text { Feasibility } \\
\text { study with } \\
\text { RCT design }\end{array}$ & $\begin{array}{l}\text { To examine } \\
\text { the feasibility } \\
\text { of problem- } \\
\text { solving } \\
\text { therapy for } \\
\text { family } \\
\text { caregivers of } \\
\text { cancer } \\
\text { patients } \\
\text { receiving } \\
\text { outpatient } \\
\text { palliative care } \\
\text { To investigate } \\
\text { the impact of } \\
\text { problem- } \\
\text { solving } \\
\text { therapy on } \\
\text { family } \\
\text { caregivers' } \\
\text { anxiety, } \\
\text { depression, } \\
\text { and quality of } \\
\text { life }\end{array}$ & $\begin{array}{l}\text { Caregivers of } \\
\text { cancer patients } \\
\text { receiving outpa- } \\
\text { tient palliative } \\
\text { care } \\
\mathrm{N}=83 \\
\mathrm{IG}= \\
\mathrm{CG}=\end{array}$ & $\begin{array}{l}\text { Individual } \\
\text { Videoconfe- } \\
\text { rencing or } \\
\text { telephone }\end{array}$ & $\begin{array}{l}3 \text { sessions } \\
\text { Duration: } \\
\text { 18days } \\
\text { Session } \\
\text { duration: } 45 \mathrm{~min}\end{array}$ & $\begin{array}{l}\text { Outcome measure- } \\
\text { ment: } \\
\text { - Anxiety } \\
\text { - QOL } \\
\text { - Depression }\end{array}$ & $\begin{array}{l}\text { - } \text { Caregiver in IG } \\
\text { reported less anxiety }\end{array}$ \\
\hline 6 & $\begin{array}{l}{[39]} \\
\text { Iran }\end{array}$ & $\begin{array}{c}\text { RCT } \\
\text { Two-group }\end{array}$ & $\begin{array}{l}\text { To determine } \\
\text { the effectiveness } \\
\text { of a theory-of- } \\
\text { planned- } \\
\text { behavior- } \\
\text { based problem- } \\
\text { solving training } \\
\text { program on the } \\
\text { coping styles of } \\
\text { family } \\
\text { caregivers of } \\
\text { psychiatric } \\
\text { inpatients. }\end{array}$ & $\begin{array}{l}\text { Family } \\
\text { caregivers of } \\
\text { - psychiatric } \\
\text { ginpatients. } \\
\text { e N }=72 \\
\text { f IG }=36 \\
\text { CG }=36\end{array}$ & $\begin{array}{l}\text { Face-to-face } \\
\text { group }\end{array}$ & $\begin{array}{l}8 \text { sessions } \\
\text { Duration: } 4 \\
\text { weeks } \\
\text { Session } \\
\text { duration: }\end{array}$ & $\begin{array}{l}\text { Timing: } \\
\text { - Preintervention } \\
\text { - Postintervention } \\
\text { immediately } \\
\text { - I-month follow-up } \\
\text { Outcome } \\
\text { measurement: } \\
\text { - Coping styles }\end{array}$ & $\begin{array}{l}\text { Immediately after } \\
\text { the intervention, IG } \\
\text { earned significantly } \\
\text { higher task-oriented } \\
\text { coping style scores } \\
\text { than CG. } \\
\text { - At 1-month } \\
\text { follow-up, IG earned } \\
\text { significantly higher } \\
\text { scores than the } \\
\text { control group for } \\
\text { task-oriented) and } \\
\text { emotion-oriented } \\
\text { coping styles. }\end{array}$ \\
\hline 7 & $\begin{array}{c}{[16]} \\
\text { Germany }\end{array}$ & $\begin{array}{c}\text { Cluster RCT } \\
\text { protocol }\end{array}$ & $\begin{array}{l}\text { To train care } \\
\text { counsellors of } \\
\text { statutory } \\
\text { long-term care } \\
\text { insurances in } \\
\text { problem- } \\
\text { solving } \\
\text { And to } \\
\text { evaluate this } \\
\text { approach as } \\
\text { an additional } \\
\text { component in } \\
\text { the statutory } \\
\text { care } \\
\text { counselling in } \\
\text { Germany. }\end{array}$ & $\begin{array}{l}\text { Distressed } \\
\text { Informal } \\
\text { caregiver } \\
38 \text { sites } \\
58 \text { care } \\
\text { counsellors }\end{array}$ & $\begin{array}{l}\text { Face-to-face } \\
\text { and telephone }\end{array}$ & $\begin{array}{l}\text { One session and } \\
\text { at least one } \\
\text { telephone } \\
\text { contact }\end{array}$ & $\begin{array}{l}\text { Timing: } \\
\text { - Preintervention } \\
\text { Outcome } \\
\text { measurement: } \\
\text { - Depression } \\
\text { - Sense of competence }\end{array}$ & - Not applicable \\
\hline
\end{tabular}




\begin{tabular}{|c|c|c|c|c|c|c|c|c|}
\hline 8 & $\begin{array}{l}{[40]} \\
\text { USA }\end{array}$ & $\begin{array}{c}\text { RCT } \\
\text { Assessment } \\
\text { blinded }\end{array}$ & $\begin{array}{l}\text { To determine } \\
\text { whether a } \\
\text { telephone- } \\
\text { based, } \\
\text { individualized } \\
\text { education and } \\
\text { mentored } \\
\text { problem- } \\
\text { solving } \\
\text { intervention } \\
\text { would improve } \\
\text { outcomes for } \\
\text { caregivers of } \\
\text { persons with } \\
\text { traumatic brain } \\
\text { injury }\end{array}$ & $\begin{array}{l}\text { Caregivers of } \\
\text { persons with } \\
\text { traumatic brain } \\
\text { injury } \\
\mathrm{N}=153 \\
\mathrm{IG}=77 \\
\mathrm{CG}=76\end{array}$ & $\begin{array}{l}\text { Telephone } \\
\text { Individual }\end{array}$ & $\begin{array}{l}\text { Maximum } 10 \\
\text { calls } \\
\text { 2-week intervals }\end{array}$ & $\begin{array}{l}\text { Timing: } \\
\text { - Preintervention } \\
\text { - 6-months post first } \\
\text { assessment } \\
\text { Outcome } \\
\text { measurement: } \\
\text { - QOL } \\
\text { - Social significance } \\
\text { - Caregiver knowledge } \\
\text { and skill acquisition }\end{array}$ & $\begin{array}{l}\text { Caregivers in IG } \\
\text { have greater social } \\
\text { problem-solving } \\
\text { abilities than CG. }\end{array}$ \\
\hline 9 & $\begin{array}{l}{[41]} \\
\text { USA }\end{array}$ & RCT & $\begin{array}{l}\text { To examine a } \\
\text { tailored, } \\
\text { problem- } \\
\text { solving } \\
\text { intervention } \\
\text { designed to } \\
\text { maximize } \\
\text { medication } \\
\text { management } \\
\text { practices } \\
\text { among } \\
\text { care-givers of } \\
\text { persons with } \\
\text { memory loss. }\end{array}$ & $\begin{array}{l}\text { Caregivers of } \\
\text { community- } \\
\text { dwelling persons } \\
\text { with memory } \\
\text { loss (Dementia) } \\
\mathrm{N}=83 \\
\mathrm{IG}=42 \\
\mathrm{CG}=41\end{array}$ & $\begin{array}{l}\text { Face-to-face \& } \\
\text { telephone } \\
\text { Individual }\end{array}$ & $\begin{array}{l}\text { - } 2 \text { or } 3 \text { home } \\
\text { visits, } 2 \\
\text { weeks apart, } \\
\text { Followed by } 2 \\
\text { - } 3 \text { telephone } \\
\text { sessions, } 7 \text { - } \\
10 \text { days apart. }\end{array}$ & $\begin{array}{l}\text { Timing: } \\
\text { - Preintervention } \\
\text { - 2-month } \\
\text { postintervention } \\
\text { Outcome } \\
\text { measurement: } \\
\text { - Medication } \\
\text { management }\end{array}$ & $\begin{array}{l}\text { - Reduced medication } \\
\text { deficiencies in both } \\
\text { groups }\end{array}$ \\
\hline 10 & $\begin{array}{l}{[26]} \\
\text { USA }\end{array}$ & $\begin{array}{c}\text { RCT } \\
\text { Assessor } \\
\text { blinded }\end{array}$ & $\begin{array}{l}\text { To determine } \\
\text { the effect of } \\
\text { early versus } \\
\text { delayed } \\
\text { initiation of a } \\
\text { palliative care } \\
\text { intervention for } \\
\text { family } \\
\text { caregivers (cgs) } \\
\text { of patients with } \\
\text { advanced } \\
\text { cancer }\end{array}$ & $\begin{array}{l}\text { Family caregiv- } \\
\text { ers of persons } \\
\text { with advanced } \\
\text { cancer } \\
\mathrm{N}=122 \\
\text { IG-early }=61 \\
\text { IG-delayed }=61\end{array}$ & $\begin{array}{l}\text { Telephone } \\
\text { Individual }\end{array}$ & $\begin{array}{l}\text { - ENABLE } \\
\text { intervention } \\
\text { - Session } \\
\text { duration; 23 } \\
\text { minutes on } \\
\text { average } \\
\text { - Duration: } 3 \\
\text { months } \\
\text { - } 3 \text { weekly } \\
\text { couch } \\
\text { sessions \& } \\
\text { monthly } \\
\text { phone call }\end{array}$ & $\begin{array}{l}\text { Timing: } \\
\text { - Preintervention } \\
\text { - Every } 6 \text { weeks until } \\
\text { week } 24 \\
\text { - Every } 3 \text { months } \\
\text { thereafter until the } \\
\text { patient participant's } \\
\text { death or study } \\
\text { completion. } \\
\text { Outcome } \\
\text { measurement: } \\
\text { - QOL } \\
\text { - Depressed mood } \\
\text { - Caregiver burden }\end{array}$ & $\begin{array}{l}\text { Early-group cgshad } \\
\text { lower depression } \\
\text { scores at } 3 \text { months } \\
\text { and lower } \\
\text { depression and } \\
\text { stress burden in the } \\
\text { terminal decline } \\
\text { analysis }\end{array}$ \\
\hline $11 / 1$ & $\begin{array}{c}{[42]} \\
\text { Hong Kong }\end{array}$ & $\begin{array}{c}\text { RCT } \\
\text { Assessor } \\
\text { blinded }\end{array}$ & $\begin{array}{l}\text { To test the } \\
\text { effects of } \\
\text { guided } \\
\text { problem- } \\
\text { solving-based } \\
\text { manual-guided } \\
\text { self-learning } \\
\text { programme for } \\
\text { family } \\
\text { caregivers } \\
\text { compared with } \\
\text { those in usual } \\
\text { family support } \\
\text { service. }\end{array}$ & $\begin{array}{l}\text { Family } \\
\text { caregivers of } \\
\text { adults with } \\
\text { recent-onset } \\
\text { psychosis } \\
\mathrm{N}=116 \\
\mathrm{IG}=58 \\
\mathrm{CG}=58\end{array}$ & $\begin{array}{l}\text { Face-to-face \& } \\
\text { telephone }\end{array}$ & $\begin{array}{l}\text { - } \text { IG } \\
\text { - Intervention } \\
\text { duration: } 5 \\
\text { months } \\
\text { - Sessions: } 5 \\
\text { assisted } \\
\text { sessions and } \\
5 \text { self-learned } \\
\text { modules } \\
\text { - Session } \\
\text { duration: } 1.5 \\
\text { - } 2 \text { hours }\end{array}$ & $\begin{array}{l}\text { Timing: } \\
\text { - Preintervention } \\
\text { - One-week } \\
\text { post-intervention } \\
\text { - 6-month } \\
\text { post-intervention } \\
\text { Caregiver Outcome } \\
\text { measurement: } \\
\text { - Caregiver burden } \\
\text { - Caregiving } \\
\text { experience } \\
\text { - Problem-solving } \\
\text { ability }\end{array}$ & $\begin{array}{l}\text { Post-intervention, } \\
\text { IG provide } \\
\text { medium-term } \\
\text { benefits to caregiver } \\
\text { burden, caregiving } \\
\text { experience and } \\
\text { patients' psychotic } \\
\text { symptoms }\end{array}$ \\
\hline
\end{tabular}




\section{Continued}

\begin{tabular}{|c|c|c|c|c|c|c|c|c|}
\hline $11 / 2$ & [43] & As above & As above & As above & As above & As above & $\begin{array}{l}\text { Timing: } \\
\text { - Preintervention } \\
\text { - One-week } \\
\text { post-intervention } \\
\text { - 6-month } \\
\text { post-intervention }\end{array}$ & $\begin{array}{l}\text { - Intervention } \\
\text { significantly } \\
\text { improved family } \\
\text { burden and } \\
\text { caregiving experience, } \\
\text { and reductions in } \\
\text { severity of psychotic } \\
\text { symptoms and } \\
\text { duration of } \\
\text { re-hospitalizations } \\
\text { than CG at 6- and } \\
\text { 12-month follow-up }\end{array}$ \\
\hline 12 & $\begin{array}{c}{[18]} \\
\text { Canada }\end{array}$ & $\begin{array}{l}\text { Matched, } \\
\text { cohort study } \\
\text { Assessor } \\
\text { blinded }\end{array}$ & $\begin{array}{l}\text { To evaluate the } \\
\text { effectiveness of } \\
\text { problem- } \\
\text { solving } \\
\text { techniques- } \\
\text { based } \\
\text { intervention } \\
\text { based in } \\
\text { enhancing } \\
\text { carers' physical } \\
\text { and emotional } \\
\text { capacity to care } \\
\text { for relatives } \\
\text { with dementia }\end{array}$ & $\begin{array}{l}\text { Informal carers } \\
\text { of patients with } \\
\text { dementia } \\
\text { receiving } \\
\text { in-home care } \\
\mathrm{N}=56 \\
\mathrm{IG}=28 \\
\mathrm{CG}=28\end{array}$ & $\begin{array}{l}\text { Individual } \\
\text { Face-to-face }\end{array}$ & $\begin{array}{l}\text { - IG } \\
\text { - } 3 \text { sessions } \\
\text { - Session } \\
\text { duration: } 1 \mathrm{~h} \\
\text { - Session } \\
\text { interval: } 1 \\
\text { week } \\
\text { Intervention } \\
\text { duration: } 3 \text { - } \\
\text { 4 weeks }\end{array}$ & $\begin{array}{l}\text { Timing: } \\
\text { - Preintervention } \\
\text { - Post-intervention } \\
\text { Caregiver Outcome } \\
\text { measurement: } \\
\text { - Coping styles } \\
\text { - Self-mastery } \\
\text { - Caregiving } \\
\text { competency } \\
\text { - Perceived stress } \\
\text { - Caregiver burden }\end{array}$ & $\begin{array}{l}\text { - Carers in IG showed } \\
\text { significantly } \\
\text { improved } \\
\text { task-oriented } \\
\text { coping, mastery, and } \\
\text { competence and } \\
\text { significantly reduced } \\
\text { emotion-oriented } \\
\text { coping, burden and } \\
\text { stress. }\end{array}$ \\
\hline 13 & $\begin{array}{l}{[44]} \\
\text { USA }\end{array}$ & $\begin{array}{c}\text { RCT } \\
2 \text { groups }\end{array}$ & $\begin{array}{l}\text { To examine } \\
\text { the efficacy of } \\
\text { Counselor- } \\
\text { Assisted } \\
\text { Problem } \\
\text { Solving } \\
\text { (CAPS) in } \\
\text { improving } \\
\text { caregiver } \\
\text { adaptation } \\
\text { following } \\
\text { traumatic } \\
\text { brain injury }\end{array}$ & $\begin{array}{l}\text { Caregivers of } \\
\text { TBI } \\
N=132 \\
\text { IG }=65 \\
\text { CG }=67\end{array}$ & $\begin{array}{l}\text { Individual } \\
1^{\text {st }} \text { session } \\
\text { using } \\
\text { face-to-face } \\
\text { and the rest } \\
\text { ones based on } \\
\text { the internet }\end{array}$ & $\begin{array}{l}\text { - } \text { IG } \\
\text { - } \text { sessions } \\
\text { period } \\
\text { - Weekly } \\
\text { sessions for } \\
\text { the first } 2 \text { - } 3 \\
\text { weeks and } \\
\text { then biweekly } \\
\text { sessions until } \\
\text { the family } \\
\text { completed } \\
\text { the initial } \\
\text { seven core } \\
\text { sessions }\end{array}$ & $\begin{array}{l}\text { Timing: } \\
\text { - Preintervention } \\
\text { - Post-intervention } \\
\text { 6-month post } \\
\text { baseline assessment } \\
\text { - Caregiver Outcome } \\
\text { measurement: } \\
\text { - Distress and } \\
\text { depression } \\
\text { Efficacy }\end{array}$ & $\begin{array}{l}\text { - Greater } \\
\text { improvements in } \\
\text { depression in IG } \\
\text { - No difference on } \\
\text { global distress } \\
\text { between two groups } \\
\text { - Among } \\
\text { non-frequent } \\
\text { computer users, } \\
\text { caregivers in IG } \\
\text { reported } \\
\text { significantly higher } \\
\text { levels of caregiver } \\
\text { efficacy than CG }\end{array}$ \\
\hline $14 / 1$ & $\begin{array}{c}{[31]} \\
\text { Germany }\end{array}$ & RCT & $\begin{array}{l}\text { To examine } \\
\text { the } \\
\text { effectiveness } \\
\text { of a problem- } \\
\text { solving } \\
\text { intervention } \\
\text { (PSI) for } \\
\text { stroke care- } \\
\text { givers }\end{array}$ & $\begin{array}{l}\text { Stroke caregivers } \\
\text { who provided } \\
\text { care for at least } 6 \\
\text { months and who } \\
\text { experienced } \\
\text { significant strain } \\
\text { in their role } \\
\mathrm{N}=122 \\
\mathrm{IG}=60 \\
\mathrm{CG}=62\end{array}$ & $\begin{array}{l}\text { Telephone and } \\
\text { face-to-face } \\
\text { Individual }\end{array}$ & $\begin{array}{l}\text { - } \text { IG } \\
\text { Part 1: 5home } \\
\text { visit }+4 \\
\text { telephone } \\
\text { Part 2:1 home } \\
\text { visit }+12 \\
\text { telephone } \\
\text { Delivered } \\
\text { over a } \\
\text { 3-month } \\
\text { intensive } \\
\text { intervention } \\
\text { and a } \\
\text { 9-month } \\
\text { maintenance } \\
\text { period }\end{array}$ & $\begin{array}{l}\text { Timing: } \\
\text { - Preintervention (T0) } \\
\text { - Post-intervention } \\
\text { (T1, } 3 \text { months) } \\
\text { - Post maintenance } \\
\text { period (T2; } 12 \\
\text { months) } \\
\text { Caregiver Outcome } \\
\text { measurement: } \\
\text { - Depression } \\
\text { - Caregiver mastery } \\
\text { - Problem-solving } \\
\text { ability } \\
\text { - Physical complaints } \\
\text { - Satisfaction with } \\
\text { leisure time }\end{array}$ & $\begin{array}{l}\text { - IG showed } \\
\text { significantly lower } \\
\text { levels of depressive } \\
\text { symptoms after } 3 \\
\text { months and after } 12 \\
\text { months }\end{array}$ \\
\hline
\end{tabular}


Continued

\begin{tabular}{|c|c|c|c|c|c|c|c|c|}
\hline $14 / 2$ & {$[45]$} & As above & As above & As above & As above & As above & As above & As above \\
\hline 15 & $\begin{array}{l}{[17]} \\
\text { USA }\end{array}$ & Pilot study & $\begin{array}{l}\text { To adapt } \\
\text { Problem- } \\
\text { Solving Skills } \\
\text { Training, to } \\
\text { the population } \\
\text { of caregivers } \\
\text { of youth with } \\
\text { chronic pain } \\
\text { Tested in a } \\
\text { small group of } \\
\text { parents to } \\
\text { evaluate } \\
\text { feasibility }\end{array}$ & $\begin{array}{l}\text { Parent } \\
\text { Caregivers of } \\
\text { Youth with } \\
\text { Chronic Pain } \\
6 \text { mothers of } \\
\text { youth }\end{array}$ & $\begin{array}{l}\text { Individual } \\
\text { Face-to-face }\end{array}$ & $\begin{array}{l}\text { - } 6-8 \text { sessions } \\
\text { - } 1 \text { hour each } \\
\text { - For } 8 \text { weeks }\end{array}$ & $\begin{array}{l}\text { Timing: } \\
\text { - Preintervention } \\
\text { - Post-intervention } \\
\text { Caregiver Outcome } \\
\text { measurement: } \\
\text { - Depression } \\
\text { - Mood disturbance } \\
\text { - Pain catastrophizing } \\
\text { - Parenting stress } \\
\text { - Miscarried helping } \\
\text { - Problem-solving } \\
\text { - skills } \\
\text { - Impact of chronic } \\
\text { pain }\end{array}$ & $\begin{array}{l}\text { - Parental problem- } \\
\text { solving skills } \\
\text { improved } \\
\text { - Parenting stress } \\
\text { decreased } \\
\text { - Depressive } \\
\text { symptoms decreased } \\
\text { - Mood disturbance } \\
\text { declined } \\
\text { - Parent-reported } \\
\text { miscarried helping } \\
\text { decreased } \\
\text { - Parents' catastrophic } \\
\text { thinking about their } \\
\text { child's pain declined } \\
\text { - Parents' maladaptive } \\
\text { behavioral responses } \\
\text { to their child's pain } \\
\text { also declined }\end{array}$ \\
\hline 16 & $\begin{array}{l}{[46]} \\
\text { USA }\end{array}$ & RCT & $\begin{array}{l}\text { To evaluate } \\
\text { the mental } \\
\text { health effects } \\
\text { of problem- } \\
\text { solving } \\
\text { therapy (PST), } \\
\text { for caregivers }\end{array}$ & $\begin{array}{l}\text { Caregivers of } \\
\text { individuals with } \\
\text { a recent } \\
\text { diagnosis of } \\
\text { Mild Cognitive } \\
\text { Impairment } \\
\text { (MCI) or early } \\
\text {, dementia. } \\
\mathrm{N}=73 \\
\mathrm{IG}= \\
\mathrm{CG}=\end{array}$ & $\begin{array}{l}\text { Face-to-face } \\
\text { Reinforce by } \\
\text { telephone } \\
\text { Individual }\end{array}$ & $\begin{array}{l}\text { - Phase 1: six } \\
\text { sessions } \\
\text { conducted in } \\
\text { the } \\
\text { caregiver's } \\
\text { home } \\
\text { approximately } \\
2 \text { weeks } \\
\text { apart, each } \\
\text { lasting } \\
\text { approximately } \\
\text { 1.5 hours. } \\
\text { - Phase 2: three } \\
\text { telephone } \\
\text { contacts } \\
\text { (approximately } \\
2 \text { weeks } \\
\text { apart), each } \\
\text { lasting } \\
\text { approximately } \\
45 \text { minutes. }\end{array}$ & $\begin{array}{l}\text { Timing: } \\
\text { - Preintervention } \\
\text { - } 1,3,6,12 \text { months } \\
\text { Post-intervention } \\
\text { Caregiver Outcome } \\
\text { measurement: } \\
\text { - Depression } \\
\text { - Anxiety } \\
\text { - Problem-solving } \\
\text { orientation } \\
\end{array}$ & $\begin{array}{l}\text { - IG led to } \\
\text { significantly reduced } \\
\text { depression } \\
\text { symptoms, } \\
\text { particularly among } \\
\text { early dementia } \\
\text { caregivers. } \\
\text { - IG lowered } \\
\text { caregivers' anxiety } \\
\text { levels, and led to } \\
\text { lessening of negative } \\
\text { problem orientation. }\end{array}$ \\
\hline $17 / 1$ & $\begin{array}{l}{[28]} \\
\text { Spain }\end{array}$ & $\begin{array}{l}\text { Mixed-study } \\
\text { Experimental } \\
\text { study with } \\
\text { randomized } \\
\text { control }\end{array}$ & $\begin{array}{l}\text { To evaluate the } \\
\text { effectiveness of } \\
\text { the problem- } \\
\text { solving } \\
\text { technique in } \\
\text { reducing } \\
\text { symptoms of } \\
\text { anxiety and } \\
\text { depression } \\
\text { among } \\
\text { primary } \\
\text { caregivers }\end{array}$ & $\begin{array}{l}\text { Female primary } \\
\text { caregivers of the } \\
\text { chronically ill } \\
\text { patients, } \\
\mathrm{N}=122 \\
\mathrm{IG}=61 \\
\mathrm{CG}=61\end{array}$ & $\begin{array}{l}\text { Face-to-face } \\
\text { home visit } \\
\text { Individual }\end{array}$ & - 4 sessions & $\begin{array}{l}\text { Timing: } \\
\text { - Preintervention } \\
\text { - } 1 \text {-month post } \\
\quad \text { intervention } \\
\text { Caregiver Outcome } \\
\text { measurement: } \\
\text { - Depression } \\
\text { - Anxiety } \\
\text { - Problem-solving } \\
\quad \text { orientation }\end{array}$ & $\begin{array}{l}\text { Caregivers in IG } \\
\text { reduced symptoms } \\
\text { of anxiety and } \\
\text { depression in family } \\
\text { caregivers of the } \\
\text { chronically ill } \\
\text { patients }\end{array}$ \\
\hline $17 / 2$ & {$[27]$} & As above & As above & As above & As above & As above & As above & As above \\
\hline
\end{tabular}




\begin{tabular}{|c|c|c|c|c|c|c|c|c|}
\hline $18 / 1$ & $\begin{array}{c}\text { [20] [21] } \\
\text { USA }\end{array}$ & $\begin{array}{l}\text { Longitudinal } \\
\text { repeated } \\
\text { measures with } \\
\text { one group }\end{array}$ & $\begin{array}{l}\text { To determine } \\
\text { the effect of } \\
\text { problem- } \\
\text { solving } \\
\text { education on } \\
\text { self-efficacy } \\
\text { and distress in } \\
\text { caregivers }\end{array}$ & $\begin{array}{l}\text { Informal } \\
\text { caregivers of } \\
\text { allogeneic } \\
\text { hematopoietic } \\
\text { stem cell } \\
\text { transplantation } \\
\text { patients } \\
\mathrm{N}=71\end{array}$ & $\begin{array}{l}\text { Face to face or } \\
\text { conference call }\end{array}$ & $\begin{array}{l}\text { - Three 1-hour } \\
\text { sessions }\end{array}$ & $\begin{array}{l}\text { Timing: } \\
\text { - Pre-hematopoietic } \\
\text { stem cell } \\
\text { transplantation } \\
\text { - Preintervention after } \\
\text { discharge } \\
\text { - } 6 \text { weeks after } \\
\text { discharge } \\
\text { Caregiver Outcome } \\
\text { measurement: } \\
\text { - Self-efficacy } \\
\text { - Healthy behaviors } \\
\text { - Sleep quality } \\
\text { - Fatigue } \\
\text { - Mutuality }\end{array}$ & $\begin{array}{l}\text { Active caregivers } \\
\text { reported } \\
\text { improvements in } \\
\text { self-efficacy and } \\
\text { distress post } \\
\text { problem-solving } \\
\text { education; } \\
\text { Caregiver } \\
\text { responders also } \\
\text { reported better } \\
\text { health outcomes } \\
\text { such as fatigue. }\end{array}$ \\
\hline $18 / 2$ & [19] & As above & As above & As above & As above & As above & As above & As above \\
\hline $18 / 3$ & [20] & As above & As above & As above & As above & As above & As above & As above \\
\hline 19 & $\begin{array}{l}{[33]} \\
\text { Iran }\end{array}$ & RCT & $\begin{array}{l}\text { To determine } \\
\text { the effect of a } \\
\text { supportive } \\
\text { educational } \\
\text { program, } \\
\text { based on } \\
\text { COPE model, } \\
\text { on individuals, } \\
\text { on the caring } \\
\text { burden and } \\
\text { quality of life in } \\
\text { the family } \\
\text { caregivers of } \\
\text { women with } \\
\text { breast cancer. }\end{array}$ & $\begin{array}{l}\text { Family } \\
\text { caregivers of } \\
\text { women with } \\
\text { breast cancer. } \\
\mathrm{N}=64 \\
\mathrm{IG}=32 \\
\mathrm{CG}=32\end{array}$ & $\begin{array}{l}\text { Telephone } \\
\text { \&face-to-face }\end{array}$ & $\begin{array}{l}\text { Two hospital } \\
\text { visits and two } \\
\text { telephone } \\
\text { sessions } \\
\text { based on } \\
\text { COPE model } \\
\text { for } 9 \text { days }\end{array}$ & $\begin{array}{l}\text { Timing: } \\
\text { - Preintervention } \\
\text { - One-month } \\
\text { post-intervention } \\
\text { Caregiver Outcome } \\
\text { measurement: } \\
\text { - Quality of life } \\
\text { - Caregiver burden }\end{array}$ & $\begin{array}{l}\text { Physical, mental, } \\
\text { spiritual, } \\
\text { environmental } \\
\text { domains and overall } \\
\text { quality of life in the } \\
\text { family caregivers } \\
\text { were significantly } \\
\text { increased in IG. } \\
\text { Significant } \\
\text { decreased caregiver } \\
\text { burden in IG } \\
\text { compared with CG. }\end{array}$ \\
\hline 20 & $\begin{array}{c}{[15]} \\
\text { Australia }\end{array}$ & $\begin{array}{c}\text { RCT with } \\
\text { randomization } \\
\text { in blocks of } 10 \\
\text { Assessor } \\
\text { blinded }\end{array}$ & $\begin{array}{l}\text { To examine } \\
\text { whether } \\
\text { completion of } \\
\text { a self-directed } \\
\text { problem- } \\
\text { solving } \\
\text { bibliotherapy } \\
\text { among carers } \\
\text { of young } \\
\text { people with } \\
\text { FEP led to a } \\
\text { better } \\
\text { experience of } \\
\text { caring, less } \\
\text { distress and } \\
\text { expressed } \\
\text { emotion, and } \\
\text { better general } \\
\text { health than } \\
\text { carers who } \\
\text { only received } \\
\text { treatment as } \\
\text { usual }\end{array}$ & $\begin{array}{l}\text { - Carers of } \\
\text { young people } \\
\text { with } \\
\text { first-episode } \\
\text { psychosis } \\
\text { - } \mathrm{N}=124 \\
\text { - } \mathrm{IG}=61 \\
\text { - } \mathrm{CG}=63\end{array}$ & Self-help & $\begin{array}{l}5 \text { weeks with } 2 \mathrm{~h} \\
\text { each module } \\
10 \text {-min } \\
\text { telephone call } \\
\text { each week from } \\
\text { a researcher to } \\
\text { determine } \\
\text { whether the } \\
\text { module had } \\
\text { been completed. }\end{array}$ & $\begin{array}{l}\text { Timing } \\
\text { - Preintervention } \\
\text { - } 6 \text { weeks follow-up } \\
\text { - } 16 \text { weeks follow-up } \\
\text { Caregiver outcome } \\
\text { measurement } \\
\text { - Caregiver experience } \\
\text { - Distress } \\
\text { - Expressed emotion } \\
\text { - QOL }\end{array}$ & $\begin{array}{l}\text { Caregivers in IG } \\
\text { experienced a } \\
\text { greater reduction in } \\
\text { negative emotional } \\
\text { evaluations of the } \\
\text { need to provide help } \\
\text { than CG by week } 6 \text {. } \\
\text { - Caregivers in IG } \\
\text { experience a greater } \\
\text { decrease in distress } \\
\text { from baseline to } 6 \\
\text { weeks }\end{array}$ \\
\hline
\end{tabular}




\begin{tabular}{|c|c|c|c|c|c|c|c|c|}
\hline 21 & $\begin{array}{l}{[36]} \\
\text { USA }\end{array}$ & $\begin{array}{c}\text { RCT } \\
\text { Transactional } \\
\text { theory of stress } \\
\text { and coping } \\
\text { Assessor } \\
\text { blinded }\end{array}$ & $\begin{array}{l}\text { Determinize } \\
\text { Feasibility, } \\
\text { acceptability, } \\
\text { and effect size } \\
\text { s estimates for } \\
\text { depression, } \\
\text { burden, } \\
\text { personal } \\
\text { control, } \\
\text { And PSS. }\end{array}$ & $\begin{array}{l}\text { Caregivers of } \\
\text { children with } \\
\text { mental health } \\
\text { problems } \\
\mathrm{N}=61 \\
\mathrm{IG}=30 \\
\mathrm{CG}=31\end{array}$ & $\begin{array}{l}\text { Combination } \\
\text { of face to face } \\
\text { and telephone } \\
\text { Individua }\end{array}$ & $\begin{array}{l}\text { One 1-hour } \\
\text { face to face } \\
\text { session and } \\
\text { eight weekly } \\
\text { telephone } \\
\text { sessions }\end{array}$ & $\begin{array}{l}\text { Timing } \\
\text { - Baseline } \\
\text { - Post-intervention } \\
\text { - } 3,6 \text { months } \\
\text { post-intervention } \\
\text { Caregiver outcome } \\
\text { measurement } \\
\text { - Personal control } \\
\text { - Problem-solving } \\
\text { - Depressive } \\
\text { - Bymptoms } \\
\text { - Burden }\end{array}$ & $\begin{array}{l}\text { - Reduced burden and } \\
\text { increased personal } \\
\text { control 3-months } \\
\text { post-intervention in } \\
\text { IG for caregivers } \\
\text { with or without } \\
\text { depression }\end{array}$ \\
\hline 22 & $\begin{array}{l}{[47]} \\
\text { USA }\end{array}$ & RCT & $\begin{array}{l}\text { To examine } \\
\text { the results of } \\
\text { Teen Online } \\
\text { Problem } \\
\text { Solving } \\
\text { (TOPS), in } \\
\text { increasing } \\
\text { problem- } \\
\text { solving skills } \\
\text { and } \\
\text { decreasing } \\
\text { depressive } \\
\text { symptoms } \\
\text { and global } \\
\text { distress for } \\
\text { caregivers }\end{array}$ & $\begin{array}{l}\text { - Caregivers of } \\
\text { adolescents } \\
\text { with } \\
\text { traumatic } \\
\text { brain injury } \\
\mathrm{N}=40 \\
\mathrm{IG}=20 \\
\mathrm{CG}=21\end{array}$ & $\begin{array}{l}\text { Web } \\
\text { Face-to-face } \\
\text { Individual }\end{array}$ & $\begin{array}{l}\text { - One home } \\
\text { visit and } 9 \text { - } \\
13 \text { web-based } \\
\text { sessions } \\
\text { - Each session: } \\
45-60 \mathrm{~min}\end{array}$ & $\begin{array}{l}\text { Timing } \\
\text { - Baseline } \\
\text { - Follow-up } \\
\text { assessment ( } 7.83 \mathrm{~m} \\
\quad \text { following baseline) } \\
\text { Caregiver outcome } \\
\text { measurement } \\
\text { - Problem-solving } \\
\text { - Parental distress } \\
\text { - Depression }\end{array}$ & $\begin{array}{l}\text { - Improvements in } \\
\text { problem-solving } \\
\text { skills and depression } \\
\text { were moderated by } \\
\text { family income, with } \\
\text { caregivers of lower } \\
\text { income in IG } \\
\text { reporting greater } \\
\text { improvements } \\
\text { - Increases in } \\
\text { problem-solving } \\
\text { partially mediated } \\
\text { reductions in global } \\
\text { distress. }\end{array}$ \\
\hline $23 / 1$ & $\begin{array}{l}{[48]} \\
\text { USA }\end{array}$ & $\begin{array}{c}\text { RCT } \\
\text { Stress and } \\
\text { coping model } \\
\text { Assessor } \\
\text { blinded }\end{array}$ & $\begin{array}{l}\text { To assess the } \\
\text { efficacy of a } \\
\text { caregiver } \\
\text { problem- } \\
\text { solving } \\
\text { intervention } \\
\text { (CPSI) on } \\
\text { stroke } \\
\text { caregiver } \\
\text { physical and } \\
\text { psychosocial } \\
\text { adaptation } \\
\text { compared } \\
\text { with a } \\
\text { wait-list } \\
\text { control } \\
\text { (WLC) } \\
\text { treatment, } \\
\text { And to } \\
\text { assess the } \\
\text { mediation } \\
\text { effects of } \\
\text { coping on } \\
\text { outcomes. }\end{array}$ & $\begin{array}{l}\text { Caregivers of } \\
\text { stroke } \\
\mathrm{N}(\text { baseline })= \\
255 \\
\mathrm{IG}=136 \\
\mathrm{CG}=119\end{array}$ & $\begin{array}{l}\text { Combination } \\
\text { of face to face } \\
\text { and telephone } \\
\text { Individual }\end{array}$ & $\begin{array}{l}\text { - IG } \\
\text { - } 10 \text { sessions } \\
\text { - }\end{array}$ & $\begin{array}{l}\text { Timing } \\
\text { - Baseline } \\
\text { - Postintervention } \\
\text { - } 6,12 \text { months } \\
\text { postdischarge } \\
\text { Caregiver outcome } \\
\text { measurement } \\
\text { - Outcome } \\
\text { - Depression } \\
\text { - Caregiver } \\
\text { preparedness } \\
\text { - Anxiety } \\
\text { - Family functioning } \\
\text { Moderator and } \\
\text { mediator } \\
\text { - Perceived health } \\
\text { - Resources and } \\
\text { stressors } \\
\text { - Appraisal } \\
\text { - Problem solving }\end{array}$ & $\begin{array}{l}\text { - Improved depres- } \\
\text { sion, life change, } \\
\text { and health favoring } \\
\text { IG postintervention } \\
\text { - Difference in } \\
\text { depression rate of } \\
\text { change favoring IG } \\
\text { - Perceived health, } \\
\text { threat appraisal and } \\
\text { rational } \\
\text { problem-solving } \\
\text { were significant } \\
\text { mediators for } \\
\text { outcomes }\end{array}$ \\
\hline $23 / 2$ & {$[22]$} & Pilot study & As above & As above & As above & As above & As above & As above \\
\hline
\end{tabular}




\section{Continued}

[49]

USA

[50]

$24 / 2 \quad$ USA

RCT

$24 / 3$

$24 / 5$

As above

As above

25

[54] RCT

- To examine

whether an

individualized

problem-

solving

- Caregiver of

intervention persons with

provided to severe

- Family disabilities

caregivers $\quad \mathrm{N}=147$

provide $\quad \mathrm{IG}=74$

benefits to $\quad \mathrm{CG}=73$

both

caregivers and

their care

recipients.

- To examine

the

effectiveness

of an

individualized $•$ Family

problem-

caregivers of

solving

women with

intervention disabilities

provided to $-\mathrm{N}=81$

family $\quad$ IG $=38$

caregivers of $\quad$ CG $=43$

women

living with

severe

disabilities.

$\mathrm{N}=60$

As above $\quad \mathrm{IG}=30$

$\mathrm{CG}=30$

As above

As above

As above

As above

To test the

effectiveness

of an

individualized

problem-

solving

intervention

delivered in

Videoconfe-

rencing

Caregivers of

people living

with a spinal

family

cord injury

$\mathrm{N}=61$

caregivers of $\mathrm{IG}=30$

persons living $\mathrm{CG}=30$

with a spinal

cord injury

(SCI) and

Possible

contagion

effects on care

recipients.
- IG

Timing

4 home visits preintervention (at months $1, \bullet 4,8$, and 12 months

Combination

$4,8,12$ ) and of participation

8 monthly Caregiver outcome

telephone measurement

sessions - Problem-solving

- Intervention - Depression

duration: 12 Satisfaction with life

months - Health complaints

- Caregiver burden
Timing

- 4 home visits - Baseline

4,8 and 12) assessment

and 8

monthly

telephone

calls

Caregiver outcome

measurement

- Caregiver depression

- Life satisfaction

- Intervention - Caregiver health duration:12 complaints

months

- Problem-solving abilities (at months 1, • 6, 9, 12-month reported significant

decrease in

depression over time

- Caregivers in IG

displayed gains in

constructive

problem-solving

abilities and

decreases in

dysfunctional

problem-solving.
- Caregivers in IG
- Caregivers in IG

reported a

significant decrease

in depression over

time.

- Caregivers in IG

displayed an

increase in

constructive

problem-solving

styles over the year.
Videoconfe-

rence

As above
As above
As above
Videoconfe-
rence

As above

As above

As above

As above

As above

As above
As above

As above

Significant decrease in depression among caregivers in IG

- Caregivers receiving PST reported gains in social functioning over time.

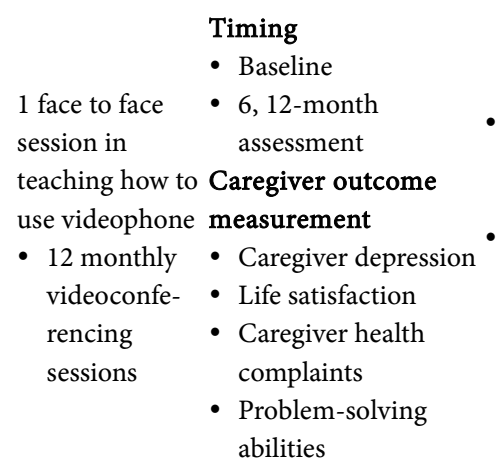




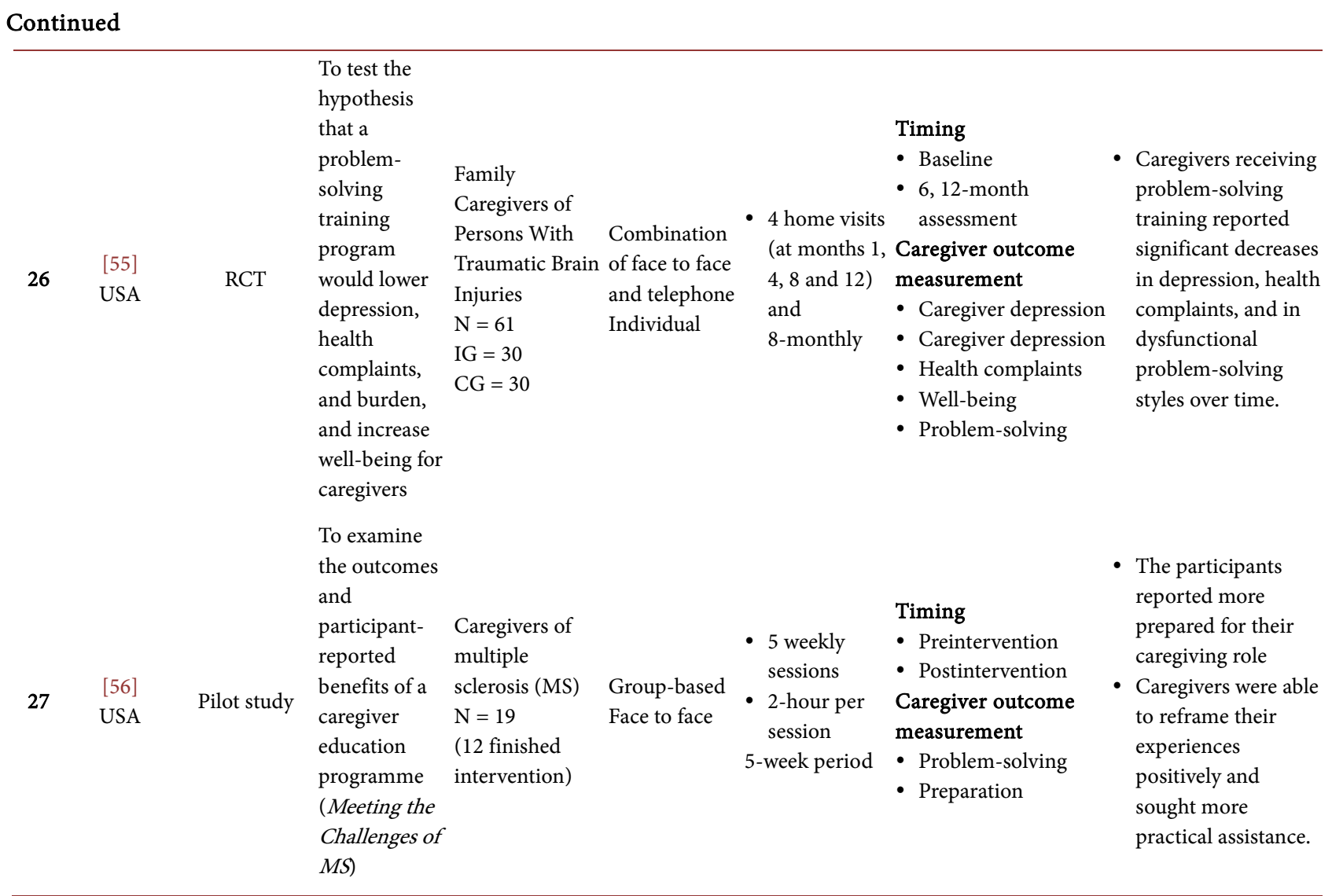

Notes: IG = intervetnion group; $\mathrm{CG}=$ control group.

\subsubsection{Problem Solving}

Some studies view problem-solving as moderator or mediator factor, while some intervention views it as outcome variable.

\section{Discussion}

As far as we know, this is the first scoping review which has endeavored to synthesize studies on problem-solving based intervention for family caregivers without focusing on one single illness of caregiving recipient. Specifically, this article described the details of each intervention design, the impact on caregiver outcomes. In total, 41 references representing 27 unique problem-solving-based intervention were included. Most interventions were targeted at caregivers of cancer, traumatic brain injury, mental illness caregivers and other conditions.

The interventions aimed to improve a wide range of caregiver outcomes by providing different delivery form, intervention duration. Of all the reported outcomes, caregiver mood, burden, quality of life and problem solving were the mostly common outcomes. Findings were mixed in terms of how problem-solving interventions impacted caregiver outcomes.

Existing research has highlighted the significance of providing support to enhance caregiver adaption [57] [58]. Of the promising interventions, problem-solving is promising in obtaining positive caregiver outcomes [9]. As com- 
pared to usual care or attention control, problem-solving intervention delivered no matter face-to-face, telephone, web or combined, have achieved enhancement in caregiver adaption. Evidence highlights the use of combination of face-to-face and telephone when delivering intervention [9] because this kind of delivery mode may be economic and has the advantage of building relationship with caregivers.

This review indicated inconsistent results among the intervention effects on caregiver outcomes. A meta-analysis would give greater power to analysis the potential effects pertaining to depression and perhaps even quality of care and burden. Meta-analysis to review the effects of problem-solving may also provide the opportunity for rigorous analysis of between-illness differences in caregiver outcomes. There are limitations in this scoping review, including the English language restriction and the start-date for the search that was set. Maybe there are some possible studies published in languages other than English and published prior to 2000 .

This study reviewed the growing body of intervention describing the details of problem-solving intervention, effects on caregiver outcomes. Most of the problem-solving intervention delivered to caregivers of dementia, traumatic brain injury, cancer, mental illness and aimed to improve a range of health outcomes for family caregivers. Problem-solving interventions were provided based on basic steps of problem-solving. Most studies reported reduced caregiver burden, decreased depression and enhanced quality although few studies did not show significant results. This review highlights that caregiver needs to be supported and problem-solving intervention may be potential to enhance caregiver experience. Future studies are wanted to employ problem-solving intervention to support caregivers.

\section{Conclusion}

This study reviewed the growing body of literature regarding problem-solving intervention delivered to caregivers. Most subjects of the problem-solving interventions are dementia caregivers, cancer caregivers. The frequently reported outcomes for caregivers are mood status, caregiver burden, quality of life and coping. The review revealed that problem-solving interventions are promising for negative outcomes of caregiving, although there lacks the effect on caregiver positive outcome, like benefit, benefit of caregiving. With studies consistently evaluating problem-solving intervention, this may be an opportune time to conduct a meta-analysis or systematic review to better-evaluate the impact of problem-solving interventions on caregiver's outcome.

\section{Conflicts of Interest}

The authors declare no conflicts of interest regarding the publication of this paper.

\section{References}

[1] Moon, M. (2017) The Unprepared Caregiver. The Gerontologist, 57, 26-31. 
https://doi.org/10.1093/geront/gnw080

[2] Mulud, Z.A. and McCarthy, G (2017) Caregiver Burden among Caregivers of Individuals with Severe Mental Illness: Testing the Moderation and Mediation Models of Resilience. Archives of Psychiatric Nursing, 31, 24-30. https://doi.org/10.1016/j.apnu.2016.07.019

[3] Lim, J., et al. (2019) Caregiver Stress in Children with Craniosynostosis: A Systematic Literature Review. Child's Nervous System, 35, 217-225. https://doi.org/10.1007/s00381-018-3959-7

[4] Torimoto-Sasai, Y., Igarashi, A., Wada, T., Ogata, Y. and Yamamoto-Mitani, N. (2015) Female Family Caregivers Face a Higher Risk of Hypertension and Lowered Estimated Glomerular Filtration Rates: A Cross-Sectional, Comparative Study. BMC Public Health, 15, Article No. 177. https://doi.org/10.1186/s12889-015-1519-6

[5] Loh, A.Z., Tana, J.S., Zhang, M.W. and Ho, R.C. (2017) The Global Prevalence of Anxiety and Depressive Symptoms among Caregivers of Stroke Survivors. Journal of the American Medical Directors Association, 18, 111-116.

https://doi.org/10.1016/j.jamda.2016.08.014

[6] Bernabeu-Mora, R., García-Guillamón, G., Montilla-Herrador, J., Escolar-Reina, P., García-Vidal, J.A. and Medina-Mirapeix, F. (2016) Rates and Predictors of Depression Status among Caregivers of Patients with COPD Hospitalized for Acute Exacerbations: A Prospective Study. International Journal of Chronic Obstructive Pulmonary Disease, 11, 3199-3205. https://doi.org/10.2147/COPD.S118109

[7] Ringer, T., Hazzan, A.A., Agarwal, A., Mutsaers, A. and Papaioannou, A. (2017) Relationship between Family Caregiver Burden and Physical Frailty in Older Adults without Dementia: A Systematic Review. Systematic Reviews, 6, Article No. 55. https://doi.org/10.1186/s13643-017-0447-1

[8] Zhu, W. and Jiang, Y. (2018) A Meta-Analytic Study of Predictors for Informal Caregiver Burden in Patients With Stroke. Journal of Stroke and Cerebrovascular Diseases, 27, 3636-3646. https://doi.org/10.1016/j.jstrokecerebrovasdis.2018.08.037

[9] Bakas, T., et al. (2014) Evidence for Stroke Family Caregiver and Dyad Interventions: A Statement for Healthcare Professionals from the American Heart Association and American Stroke Association. Stroke, 45, 2836-2852. https://doi.org/10.1161/STR.0000000000000033

[10] D’Zurilla, T.J. and Nezu, A.M. (1999) Problem-Solving Therapy: A Social Competence Approach to Clinical Intervention. Springer Publishing Company, New York.

[11] D'Zurilla, T.J. and Nezu, A.M. (2010) Problem-Solving Therapy. Handbook of Cognitive-Behavioral Therapies, 3, 197-225.

[12] Del-Pino-Casado, R., Frías-Osuna, A., Palomino-Moral, P.A. and Pancorbo-Hidalgo, P.L. (2011) Coping and Subjective Burden in Caregivers of Older Relatives: A Quantitative Systematic Review. Journal of Advanced Nursing, 67, 2311-2322. https://doi.org/10.1111/j.1365-2648.2011.05725.x

[13] Kirkham, J.G., Choi, N. and Seitz, D.P. (2016) Meta-Analysis of Problem Solving Therapy for the Treatment of Major Depressive Disorder in Older Adults. International Journal of Geriatric Psychiatry, 31, 526-535. https://doi.org/10.1002/gps.4358

[14] Arksey, H. and O’Malley, L. (2005) Scoping Studies: Towards a Methodological Framework. International Journal of Social Research Methodology, 8, 19-32. https://doi.org/10.1080/1364557032000119616

[15] McCann, T.V., et al. (2013) A Randomized Controlled Trial of Bibliotherapy for Carers of Young People with First-Episode Psychosis. Schizophrenia Bulletin, 39, 
1307-1317. https://doi.org/10.1093/schbul/sbs121

[16] Pfeiffer, K., et al. (2017) Problem-Solving in Caregiver-Counselling (PLiP Study): Study Protocol of a Cluster Randomized Pragmatic trial. BMC Geriatrics, 17, 64. https://doi.org/10.1186/s12877-017-0456-x

[17] Palermo, T.M., Law, E.F., Essner, B., Jessen-Fiddick, T. and Eccleston, C. (2014) Adaptation of Problem-Solving Skills Training (PSST) for Parent Caregivers of Youth with Chronic Pain. Clinical Practice in Pediatric Psychology, 2, 212-223. https://doi.org/10.1037/cpp0000067

[18] Chiu, M., Pauley, T., Wesson, V., Pushpakumar, D. and Sadavoy, J. (2015) Evaluation of a Problem-Solving (PS) Techniques-Based Intervention for Informal Carers of Patients with Dementia Receiving in-Home Care. International Psychogeriatrics, 27, 937-948. https://doi.org/10.1017/S1041610214002798

[19] Bevans, M., et al. (2010) An Individualized Dyadic Problem-Solving Education Intervention for Patients and Family Caregivers during Allogeneic Hematopoietic Stem Cell Transplantation: A Feasibility Study. Cancer Nursing, 33, E24-E32. https://doi.org/10.1097/NCC.0b013e3181be5e6d

[20] Bevans, M., et al. (2008) An Individualized Dyadic Problem-Solving Education Intervention (PSEI) to Improve Problem-Solving Skills of Patients and Family Caregivers during Allogeneic Hematopoietic Stem Cell Transplantation (a-HSCT). Psycho-Oncology, 17, S160-S161.

[21] Bevans, M., et al. (2014) A Problem-Solving Education Intervention in Caregivers and Patients during Allogeneic Hematopoietic Stem Cell Transplantation. Journal of Health Psychology, 19, 602-617. https://doi.org/10.1177/1359105313475902

[22] King, R.B., Hartke, R.J. and Denby, F. (2007) Problem-Solving Early Intervention: A Pilot Study of Stroke Caregivers. Rehabilitation Nursing, 32, 68-76. https://doi.org/10.1002/j.2048-7940.2007.tb00154.x

[23] Demiris, G., Oliver, D.P., Washington, K. and Pike, K. (2019) A Problem-Solving Intervention for Hospice Family Caregivers: A Randomized Clinical Trial. Journal of the American Geriatrics Society, 67, 1345-1352. https://doi.org/10.1111/jgs.15894

[24] Demiris, G., et al. (2010) A Problem Solving Intervention for Hospice Caregivers: A Pilot Study. Journal of Palliative Medicine, 13, 1005-1011. https://doi.org/10.1089/jpm.2010.0022

[25] Demiris, G., et al. (2012) A Noninferiority Trial of a Problem-Solving Intervention for Hospice Caregivers: In Person versus Videophone. Journal of Palliative Medicine, 15, 653-660. https://doi.org/10.1089/jpm.2011.0488

[26] Dionne-Odom, J.N., et al. (2015) Benefits of Early Versus Delayed Palliative Care to Informal Family Caregivers of Patients With Advanced Cancer: Outcomes from the ENABLE III Randomized Controlled Trial. Journal of Clinical Oncology, 33, 1446-1452. https://doi.org/10.1200/JCO.2014.58.7824

[27] Ferre-Grau, C., et al. (2014) Caring for Family Caregivers: An Analysis of a Family-Centered Intervention. Revista Da Escola De Enfermagem Da Usp, 48, 87-94. https://doi.org/10.1590/S0080-623420140000600013

[28] Ferre-Grau, C., et al. (2014) Effectiveness of Problem-Solving Technique in Caring for Family Caregivers: A Clinical Trial Study in an Urban Area of Catalonia (Spain). Journal of Clinical Nursing, 23, 288-295. https://doi.org/10.1111/jocn.12485

[29] Lopez, L., et al. (2019) Problem-Solving Intervention to Prevent Depression in Non-Professional Caregivers: A Randomized Controlled Trial with 8 Years of Follow-Up. Psychological Medicine, 1-8. https://doi.org/10.1017/S0033291719000916 
[30] Otero, P., Smit, F., Cuijpers, P., Torres, A., Blanco, V. and Vázquez, F.L. (2015) Long-Term Efficacy of Indicated Prevention of Depression in Non-Professional Caregivers: Randomized Controlled Trial. Psychological Medicine, 45, 1401-1412. https://doi.org/10.1017/S0033291714002505

[31] Pfeiffer, K., et al. (2014) Telephone-Based Problem-Solving Intervention for Family Caregivers of Stroke Survivors: A Randomized Controlled Trial. Journal of Consulting and Clinical Psychology, 82, 628-643. https://doi.org/10.1037/a0036987

[32] Vazquez Gonzalez, F.L., et al. (2013) A Brief Problem-Solving Indicated-Prevention Intervention for Prevention of Depression in Nonprofessional Caregivers. Psicothema, 25, 87-92.

[33] Bahrami, M. and Farzi, S. (2014) The Effect of a Supportive Educational Program Based on COPE Model on Caring Burden and Quality of Life in Family Caregivers of Women with Breast Cancer. Iranian Journal of Nursing and Midwifery Research, 19, 119-126.

[34] Dionne-Odom, J.N., Lyons, K.D., Akyar, I. and Bakitas, M.A. (2016) Coaching Family Caregivers to Become Better Problem Solvers When Caring for Persons with Advanced Cancer. Journal of Social Work in End-of-Life \& Palliative Care, 12, 63-81. https://doi.org/10.1080/15524256.2016.1156607

[35] Garand, L., et al. (2019) Problem-Solving Therapy Reduces Subjective Burden Levels in Caregivers of Family Members with Mild Cognitive Impairment or Early-Stage Dementia: Secondary Analysis of a Randomized Clinical Trial. International Journal of Geriatric Psychiatry, 34, 957-965. https://doi.org/10.1002/gps.5095

[36] Gerkensmeyer, J.E., et al. (2013) Problem-Solving Intervention for Caregivers of Children with Mental Health Problems. Archives of Psychiatric Nursing, 27, 112-120. https://doi.org/10.1016/j.apnu.2013.01.004

[37] Malcarne, V.L., Ko, C.M., Roesch, S.C., Banthia, R. and Sadler, G.R. (2019) Efficacy of Problem-Solving Therapy for Spouses of Men with Prostate Cancer: A Randomized Controlled Trial. Psycho-Oncology, 28, 497-504. https://doi.org/10.1002/pon.4964

[38] Washington, K.T., et al. (2018) Delivering Problem-Solving Therapy to Family Caregivers of People with Cancer: A Feasibility Study in Outpatient Palliative Care. Psycho-Oncology, 27, 2494-2499. https://doi.org/10.1002/pon.4859

[39] Abedi, Z., Alavi, M., Ghazavi, Z., Visentin, D. and Cleary, M. (2019) Improving Coping Styles in Family Caregivers of Psychiatric Inpatients Using Planned Behavior Problem-Solving Training. Journal of Nursing Research. https://doi.org/10.1097/jnr.0000000000000320

[40] Powell, J.M., Fraser, R., Brockway, J., Temkin, N. and Bell, K. (2016) A Telehealth Approach to Caregiver Self-Management Following Traumatic Brain Injury: A Randomized Controlled Trial. Journal of Head Trauma Rehabilitation, 31, 180-190. https://doi.org/10.1097/HTR.0000000000000167

[41] Lingler, J.H. (2016) An Intervention to Maximize Medication Management by Caregivers of Persons with Memory Loss: Intervention Overview and Two-Month Outcomes. Geriatric Nursing, 37, 186-191.

[42] Chien, W.T., Yip, A.L.K., Liu, J.Y.W. and McMaster, T.W. (2016) The Effectiveness of Manual-Guided, Problem-Solving-Based Self-Learning Programme for Family Caregivers of People with Recent-Onset Psychosis: A Randomised Controlled Trial with 6-Month Follow-Up. International Journal of Nursing Studies, 59, 141-155. https://doi.org/10.1016/j.ijnurstu.2016.03.018

[43] Chien, W.T., Thompson, D.R., Lubman, D.I. and McCann, T.V. (2016) A Rando- 
mized Controlled Trial of Clinician-Supported Problem-Solving Bibliotherapy for Family Caregivers of People with First-Episode Psychosis. Schizophrenia Bulletin, 42, 1457-1466. https://doi.org/10.1093/schbul/sbw054

[44] Wade, S.L., et al. (2014) Counselor-Assisted Problem Solving Improves Caregiver Efficacy Following Adolescent Brain Injury. Rehabilitation Psychology, 59, 1-9. https://doi.org/10.1037/a0034911

[45] Pfeiffer, K., et al. (2011) Telephone-Based Problem Solving Therapy for Family Caregivers of Stroke Survivors-Results of a Randomized Controlled Trial. Gerontologist, 51, 596.

[46] Garand, L., et al. (2014) Effects of Problem Solving Therapy on Mental Health Outcomes in Family Caregivers of Persons with a New Diagnosis of Mild Cognitive Impairment or Early Dementia: A Randomized Controlled Trial. The American Journal of Geriatric Psychiatry, 22, 771-781.

[47] Wade, S.L., et al. (2012) A Randomized Trial of Teen Online Problem Solving: Efficacy in Improving Caregiver Outcomes after Brain Injury. Health Psychology, 31, 767-776. https://doi.org/10.1037/a0028440

[48] King, R.B., et al. (2012) A Problem-Solving Early Intervention for Stroke Caregivers: One Year Follow-Up. Rehabilitation Nursing, 37, 231-243.

[49] Berry, J.W., Elliott, T.R., Grant, J.S., Edwards, G. and Fine, P.R. (2012) Does Problem-Solving Training for Family Caregivers Benefit Their Care Recipients with Severe Disabilities? A Latent Growth Model of the Project CLUES Randomized Clinical Trial. Rehabilitation Psychology, 57, 98-112. https://doi.org/10.1037/a0028229

[50] Elliott, T.R., Berry, J.W. and Grant, J.S. (2009) Problem-Solving Training for Family Caregivers of Women with Disabilities: A Randomized Clinical Trial. Behaviour Research and Therapy, 47, 548-558.

[51] Elliott, T.R. and Berry, J.W. (2009) Brief Problem-Solving Training for Family Caregivers of Persons with Recent-Onset Spinal Cord Injuries: A Randomized Controlled Trial. Journal of Clinical Psychology, 65, 406-422. https://doi.org/10.1002/jclp.20527

[52] Garand, L. (2009) Adaptation and Evaluation of Problem Solving Therapy for the Prevention of Depression in Family Caregivers of Persons with Mild Cognitive Impairment (MCI). Gerontologist, 49, 498.

[53] Grant, J.S., Elliott, T.R., Weaver, M., Bartolucci, A.A. and Giger, J.N. (2002) Telephone Intervention with Family Caregivers of Stroke Survivors after Rehabilitation. Stroke, 33, 2060-2065. https://doi.org/10.1161/01.STR.0000020711.38824.E3

[54] Timothy, R.E., Brossart, D., Berry, J.W. and Fine, P.R. (2008) Problem-Solving Training via Videoconferencing for Family Caregivers of Persons with Spinal Cord Injuries: A Randomized Controlled Trial. Behaviour Research and Therapy, 46, 1220-1229. https://doi.org/10.1016/j.brat.2008.08.004

[55] Rivera, P.A., Elliott, T.R., Berry, J.W. and Grant, J.S. (2008) Problem-Solving Training for Family Caregivers of Persons with Traumatic Brain Injuries: A Randomized Controlled Trial. Archives of Physical Medicine and Rehabilitation, 89, 931-941.

[56] Finlayson, M., Preissner, K. and Garcia, J. (2009) Pilot Study of an Educational Programme for Caregivers of People Ageing with Multiple Sclerosis. British Journal of Occupational Therapy, 72, 11-19. https://doi.org/10.1177/030802260907200104

[57] Thomas, S., Dalton, J., Harden, M., Eastwood, A. and Parker, G. (2017) Updated Meta-Review of Evidence on Support for Carers, in Updated Meta-Review of Evi- 
dence on Support for Carers. Health Services and Delivery Research, 5.

https://doi.org/10.3310/hsdr05120

[58] Bakas, T., McCarthy, M. and Miller, E.T. (2017) Update on the State of the Evidence for Stroke Family Caregiver and Dyad Interventions. Stroke, 48, e122-e125.

https://doi.org/10.1161/STROKEAHA.117.016052 